\title{
The Impact of Porosity upon the Cathodoluminescence from III-V Compounds
}

\author{
M.A. Stevens-Kalceff,* I.M. Tiginyanu,** S. Langa*** and H. Föll.***
}

*School of Physics and Electron Microscope Unit. University of New South Wales, Sydney NSW 2052 Australia.

**Laboratory of Low-Dimensional Semiconductor Structures, Institute of Applied Physics, Technical University of Moldova, MD-2004 Chisinau, Moldova.

***Materials Science Department, Faculty of Engineering, Christian-Albrechts University, Kaiserstr. 2, 24143 Kiel, Germany.

Porosity is an effective tool for engineering fundamental properties of semiconductor materials including the band gap, band structure, phonon spectrum, refractive indices, resistivity, thermal conductivity, etc. Nano-porous $\mathrm{Si}$ exhibits luminescence at wavelengths dependent on the dimensions of the porous skeleton. [1] More recently, anodic etching techniques have been used for fabricating porous structures in III-V compounds. [2] Compared with porous silicon, III-V materials have a number of important advantages related to their chemical composition and acentric properties. In particular, porous III-V compounds exhibit Fröhlich-type surface-related vibrations with porositytunable frequencies and efficient optical second harmonic generation. [3,4] The enhanced non-linear optical response and luminescence from porous III-V compounds may enable the development of a fully integrated light source and frequency converter sub-systems. In addition, the anisotropy of III-V materials with respect to chemical etching may also enable the production of ordered arrays of pores with the possibility of 2 and 3 dimensional structures for photonic applications including Bragg mirrors, Fabry Perot filters and waveguides, etc. These applications/ device structures may be able to be integrated into optoelectronic circuits.

The development of applications involving porous materials requires understanding of their special properties. Most published investigations have used photoluminescence (PL) techniques to determine the average emission characteristics of porous III-V materials. In this work, Cathodoluminescence Microanalysis techniques show that the morphology and emission microcharacteristics of porous III-V materials (Indium and Gallium Phosphides in particular), are highly correlated, and demonstrate that the spatial distribution of emission can be controlled. [5]

For example, FIG. 1 shows cross sectional secondary electron and panchromatic CL images from porous InP, which has been anodized by periodically switching the current between $0 \mathrm{~mA}$ for $6 \mathrm{~s}$ and $80 \mathrm{~mA}$ for $6 \mathrm{~s}$. The insert shows the secondary electron image of the interface between two neighboring porous layers. Synchronous spatial modulation of porosity and Cathodoluminescence (CL) intensity is achieved in InP by periodically switching the dissolution current. Pore growth ceases during the interruption to the etching process. A new nucleation phase is formed, and pore growth is re-established when current is reapplied. Note that in this case higher porosity is associated with lower CL emission efficiency. Luminescent emission is attenuated from unpassivated pore surfaces due to the presence of non-radiative recombination centers. The quenching increases as the porosity increases (i.e. the dimensions of the porous skeleton decrease) and therefore the surface to volume ratio increases. Oxygen is known to passivate InP surfaces, however the HCL electrolyte used in this case to process the InP specimen removes oxides from the InP pore surfaces. 
In summary, this work shows that luminescence intensity and porosity of III-V compounds can be spatially modulated by varying the anodization conditions, including the dissolution current, applied voltage, and type and concentration of anions in the electrolyte. Synchronous spatial modulation of porosity and luminescence intensity can be achieved by periodically switching the dissolution current, by varying the applied voltage and by self induced correlated voltage oscillations related to high pore density and interactions between neighboring pores. Emission from porous materials is strongly influenced by surface effects (e.g. radiative versus non-radiative processes) and may also be influenced by quantum confinement effects depending on the dimensions of the porous skeleton of the particular material.

\section{References}

[1] A.G. Cullis et al J. Appl. Phys. 82 (1997) 909.

[2] I.M. Tiginyanu et al., Appl. Phys. Lett.71 (1997) 3829.

[3] I.M. Tiginyanu et al., Phys. Rev. B 55 (1997) 6739.

[4] I.M. Tiginyanu et al., Appl. Phys. Lett. 77 (2000) 2415.

[5] M. A. Stevens-Kalceff et al., J. Appl. Phys. 89 (2001) 2560.

[6] Support from the Australian Research Council, the Electron Microscope Unit at UNSW, W. Kalceff and Deutsche Forschungsgemeinschaft (Grant No 436 MOL 113/2/0-1) is gratefully acknowledged.

*Marion.Stevens-Kalceff@phys.unsw.edu.au

**tiginyan@utm.md
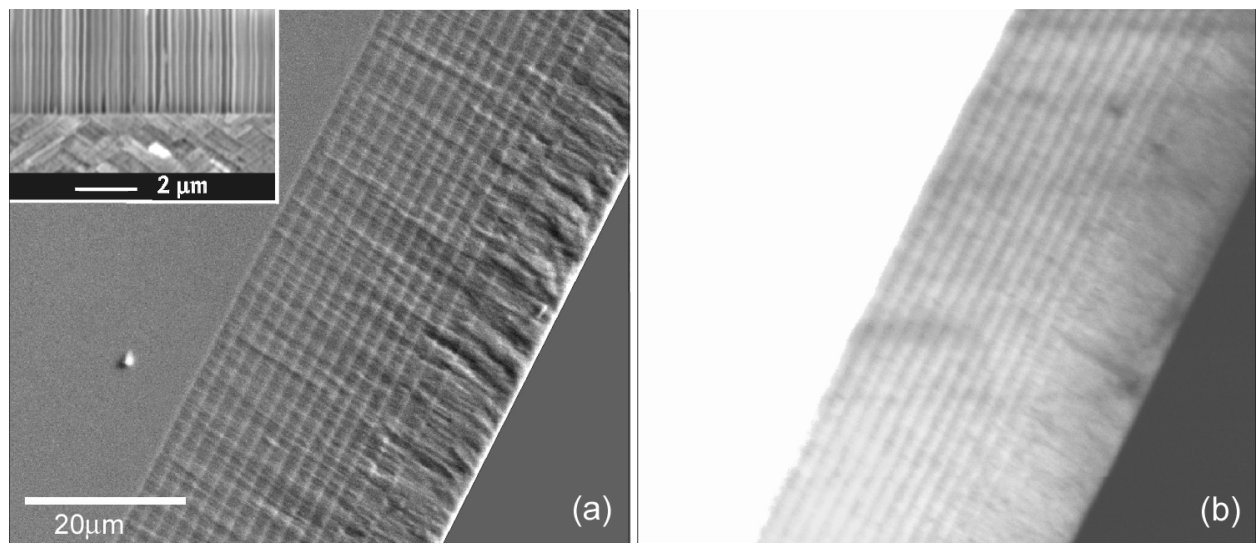

FIG. 1. Cross sectional (a) secondary electron (SE) and (b) panchromatic CL images from porous InP which has been anodized by periodically switching the current on and off. The insert shows the SEI image of the interface between two neighboring porous layers. 